\title{
A Novel Construction of Perfect Strict Avalanche Criterion S-box using Simple Irreducible Polynomials
}

\author{
Alamsyah \\ Department of Computer Science, Universitas Negeri Semarang Sekaran Campus, Gunungpati, Semarang, \\ 50229, Indonesia \\ Email: alamsyah@mail.unnes.ac.id
}

\begin{abstract}
An irreducible polynomial is one of the main components in building an S-box with an algebraic technique approach. The selection of the precise irreducible polynomial will determine the quality of the S-box produced. One method for determining good S-box quality is strict avalanche criterion will be perfect if it has a value of 0.5 . Unfortunately, in previous studies, the strict avalanche criterion value of the S-box produced still did not reach perfect value. In this paper, we will discuss S-box construction using selected irreducible polynomials. This selection is based on the number of elements of the least amount of irreducible polynomials that make it easier to construct S-box construction. There are 17 irreducible polynomials that meet these criteria. The strict avalanche criterion test results show that the irreducible polynomial $p_{17}(x)=x^{8}$ $+x^{7}+x^{6}+x+1$ is the best with a perfect SAC value of 0.5 . One indicator that a robust S-box is an ideal strict avalanche criterion value of 0.5
\end{abstract}

Keywords: Strict Avalanche Criterion, S-box, Irreducible Polynomial, Algebraic Technique

\section{INTRODUCTION}

S-box which is also known as a substitution box has a function in the process of randomizing data bits [1]. The strength of the S-box generated will determine the durability of a message that has been encrypted from linear and differential attacks [2]. The method used in constructing S-box construction in this paper is algebraic techniques. In algebraic techniques, irreducible polynomials play an important role in making S-box construction [3]. An irreducible polynomial is a polynomial that has two multiplication factors, namely itself and 1 [4]. In GF $\left(\left(2^{8}\right)\right.$, the irreducible polynomial that is built has the highest degree of 8 which is used to make a multiplicative inverse. The resulting multiplicative inverse will be used to build an S-box. Hence, the role of the irreducible polynomial is crucial in building a strong S-box construction [5].

One of the criteria for determining the strength of an S-box is the perfect value of the strict avalanche criterion (SAC) [1]. The SAC is used to see changes that occur in input bits and output bits. If there is a change in 1-bit of input, ideally there should be half of the output bit changed. This means that the perfect SAC value is 0.5 [6]. The SAC value produced by the S-boxes construction that has been carried out by previous researchers is various. Girija and Singh [7] developed S-boxes with a double random phase encoding (DRPE) system. Farwa et al. [8] built an S-box with a specific nonlinear and iterative map approach. Çavuşoğlu et al. [9] developed an S-box design by placing an 8 -bit value taken from a random number generator (RNG). 
Hussain et al. [10] built S-box construction based on quantum magnets and Lorenz chaotic system matrix. Belazi et al. [11], Özkaynak et al. [12], Özkaynak and Yavuz [13], Liu et al. [14], Khan et al. [15], and Khan and Syah [16] proposed S-boxes based on a chaotic system. Proposed S-boxes were also developed by [17], [18], [19], [20], [21], [22], and [23]. Unfortunately, the resulting SAC value [7-23] is only close to the perfect value of 0.5 .

In this paper, we will present S-boxes construction using simple irreducible polynomials. S-boxes are built based on 17 simple irreducible polynomials, i.e., $p_{1}(x)$, $p_{2}(x), \ldots, p_{17}(x)$. The resulting S-boxes will be tested using SAC. The proposed S-box is the selected S-box that has the best SAC value compared to previous studies. In the next section, we will discuss the irreducible polynomial.

\section{METHODS}

\subsection{Irreducible Polynomial}

An irreducible polynomial is a polynomial that has two multiplication factors, i.e., itself and 1 . Table 1 shows the irreducible polynomials classified according to the order of the number of the smallest polynomial elements [24] as listed in [25], [26], [27], [28], and [29]. Based on Table 1, 17 irreducible polynomials have the least number of polynomial elements, namely five elements. Through 17 selected irreducible polynomials, the multiplicative inverse will be built.

Table 1. Number Of Irreducible Polynomials Elements

\begin{tabular}{lc}
\hline Irreducible Polynomials & Number of elements \\
\hline$x^{8}+x^{4}+x^{3}+x+1$ & 5 \\
$x^{8}+x^{4}+x^{3}+x^{2}+1$ & 5 \\
$x^{8}+x^{5}+x^{3}+x+1$ & 5 \\
$x^{8}+x^{5}+x^{3}+x^{2}+1$ & 5 \\
$x^{8}+x^{5}+x^{4}+x^{3}+1$ & 5 \\
$x^{8}+x^{6}+x^{3}+x^{2}+1$ & 5 \\
$x^{8}+x^{6}+x^{5}+x+1$ & 5 \\
$x^{8}+x^{6}+x^{5}+x^{2}+1$ & 5 \\
$x^{8}+x^{6}+x^{5}+x^{3}+1$ & 5 \\
$x^{8}+x^{6}+x^{5}+x^{4}+1$ & 5 \\
$x^{8}+x^{7}+x^{2}+x+1$ & 5 \\
$x^{8}+x^{7}+x^{3}+x+1$ & 5 \\
$x^{8}+x^{7}+x^{3}+x^{2}+1$ & 5 \\
$x^{8}+x^{7}+x^{5}+x+1$ & 5 \\
$x^{8}+x^{7}+x^{5}+x^{3}+1$ & 5 \\
$x^{8}+x^{7}+x^{5}+x^{4}+1$ & 5 \\
$x^{8}+x^{7}+x^{6}+x+1$ & 5 \\
$x^{8}+x^{5}+x^{4}+x^{3}+x^{2}+x+1$ & 7 \\
$x^{8}+x^{6}+x^{4}+x^{3}+x^{2}+x+1$ & 7 \\
$x^{8}+x^{6}+x^{5}+x^{4}+x^{2}+x+1$ & 7 \\
$x^{8}+x^{6}+x^{5}+x^{4}+x^{3}+x+1$ & 7 \\
$x^{8}+x^{7}+x^{4}+x^{3}+x^{2}+x+1$ & 7
\end{tabular}




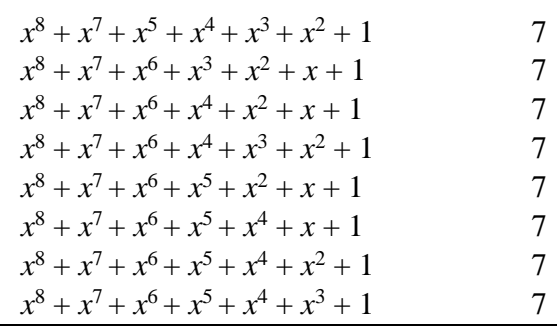

Further discussion of multiplicative inverse construction is presented in the novel Sboxes construction section.

\subsection{Novel S-Boxes Construction}

In this section, we will introduce S-box construction based on simple irreducible polynomial specifically irreducible polynomial which has the least polynomial elements. The S-box construction proposed based on the scheme in Figure 1. According to Figure 1, the S-box construction is generated from a multiplicative inverse applied to affine mapping. The affine mapping consists of an affine matrix and the addition of a constant 8-bit vector as shown in Eq. (1).

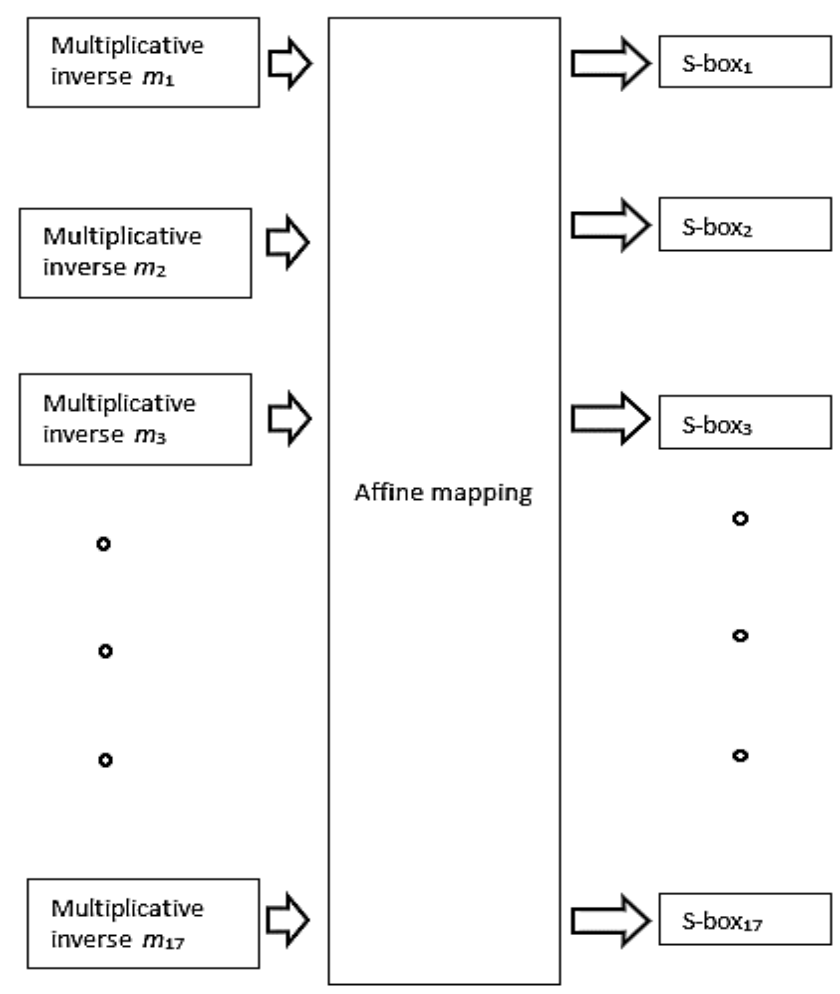

Figure 1. The construction scheme for the proposed S-boxes 


$$
\left[\begin{array}{l}
b_{0} \\
b_{1} \\
b_{2} \\
b_{3} \\
b_{4} \\
b_{5} \\
b_{6} \\
b_{7}
\end{array}\right]=\left[\begin{array}{llllllll}
1 & 0 & 0 & 0 & 1 & 1 & 1 & 1 \\
1 & 1 & 0 & 0 & 0 & 1 & 1 & 1 \\
1 & 1 & 1 & 0 & 0 & 0 & 1 & 1 \\
1 & 1 & 1 & 1 & 0 & 0 & 0 & 1 \\
1 & 1 & 1 & 1 & 1 & 0 & 0 & 0 \\
0 & 1 & 1 & 1 & 1 & 1 & 0 & 0 \\
0 & 0 & 1 & 1 & 1 & 1 & 1 & 0 \\
0 & 0 & 0 & 1 & 1 & 1 & 1 & 1
\end{array}\right]\left[\begin{array}{l}
b^{\prime}{ }_{0} \\
b^{\prime}{ }_{1} \\
b_{2}^{\prime} \\
b^{\prime}{ }_{3} \\
b_{4}^{\prime} \\
b^{\prime}{ }_{5} \\
b_{6}^{\prime} \\
b_{7}^{\prime}
\end{array}\right]+\left[\begin{array}{l}
1 \\
0 \\
0 \\
0 \\
0 \\
1 \\
1 \\
0
\end{array}\right] \bmod 2
$$

For example, $\mathrm{S}-$ box $_{1}, \mathrm{~S}-$ box $_{2}$, and S-box 3 will be built using irreducible polynomials $p_{1}(x)=x^{8}+x^{4}+x^{3}+x+1, p_{2}(x)=x^{8}+x^{4}+x^{3}+x^{2}+1$, and $p_{3}(x)=x^{8}+x^{5}+x^{3}+x+1$. Tables 2, 3 and 4 are multiplicative inverse tables of $m_{1}, m_{2}$, and $m_{3}$, respectively, which are built based on the irreducible polynomials. Each inverse multiplicative is applied to Eq. (1) so that Tables 5, 6 and 7 are obtained which are S-box $1, S-$ box $_{2}$, and S-box 3 , respectively.

Table 2. The proposed multiplicative inverse $m_{1}$

\begin{tabular}{llllllllllllllll}
\hline 0 & 1 & 141 & 246 & 203 & 82 & 123 & 209 & 232 & 79 & 41 & 192 & 176 & 225 & 229 & 199 \\
116 & 180 & 170 & 75 & 153 & 43 & 96 & 95 & 88 & 63 & 253 & 204 & 255 & 64 & 238 & 178 \\
58 & 110 & 90 & 241 & 85 & 77 & 168 & 201 & 193 & 10 & 152 & 21 & 48 & 68 & 162 & 194 \\
44 & 69 & 146 & 108 & 243 & 57 & 102 & 66 & 242 & 53 & 32 & 111 & 119 & 187 & 89 & 25 \\
29 & 254 & 55 & 103 & 45 & 49 & 245 & 105 & 167 & 100 & 171 & 19 & 84 & 37 & 233 & 9 \\
237 & 92 & 5 & 202 & 76 & 36 & 135 & 191 & 24 & 62 & 34 & 240 & 81 & 236 & 97 & 23 \\
22 & 94 & 175 & 211 & 73 & 166 & 54 & 67 & 244 & 71 & 145 & 223 & 51 & 147 & 33 & 59 \\
121 & 183 & 151 & 133 & 16 & 181 & 186 & 60 & 182 & 112 & 208 & 6 & 161 & 250 & 129 & 130 \\
131 & 126 & 127 & 128 & 150 & 115 & 190 & 86 & 155 & 158 & 149 & 217 & 247 & 2 & 185 & 164 \\
222 & 106 & 50 & 109 & 216 & 138 & 132 & 114 & 42 & 20 & 159 & 136 & 249 & 220 & 137 & 154 \\
251 & 124 & 46 & 195 & 143 & 184 & 101 & 72 & 38 & 200 & 18 & 74 & 206 & 231 & 210 & 98 \\
12 & 224 & 31 & 239 & 17 & 117 & 120 & 113 & 165 & 142 & 118 & 61 & 189 & 188 & 134 & 87 \\
11 & 40 & 47 & 163 & 218 & 212 & 228 & 15 & 169 & 39 & 83 & 4 & 27 & 252 & 172 & 230 \\
122 & 7 & 174 & 99 & 197 & 219 & 226 & 234 & 148 & 139 & 196 & 213 & 157 & 248 & 144 & 107 \\
177 & 13 & 214 & 235 & 198 & 14 & 207 & 173 & 8 & 78 & 215 & 227 & 93 & 80 & 30 & 179 \\
91 & 35 & 56 & 52 & 104 & 70 & 3 & 140 & 221 & 156 & 125 & 160 & 205 & 26 & 65 & 28 \\
\hline
\end{tabular}


Table 3. The proposed multiplicative inverse $m_{2}$

\begin{tabular}{llllllllllllllll}
\hline 0 & 1 & 142 & 244 & 71 & 167 & 122 & 186 & 173 & 157 & 221 & 152 & 61 & 170 & 93 & 150 \\
216 & 114 & 192 & 88 & 224 & 62 & 76 & 102 & 144 & 222 & 85 & 128 & 160 & 131 & 75 & 42 \\
108 & 237 & 57 & 81 & 96 & 86 & 44 & 138 & 112 & 208 & 31 & 74 & 38 & 139 & 51 & 110 \\
72 & 137 & 111 & 46 & 164 & 195 & 64 & 94 & 80 & 34 & 207 & 169 & 171 & 12 & 21 & 225 \\
54 & 95 & 248 & 213 & 146 & 78 & 166 & 4 & 48 & 136 & 43 & 30 & 22 & 103 & 69 & 147 \\
56 & 35 & 104 & 140 & 129 & 26 & 37 & 97 & 19 & 193 & 203 & 99 & 151 & 14 & 55 & 65 \\
36 & 87 & 202 & 91 & 185 & 196 & 23 & 77 & 82 & 141 & 239 & 179 & 32 & 236 & 47 & 50 \\
40 & 209 & 17 & 217 & 233 & 251 & 218 & 121 & 219 & 119 & 6 & 187 & 132 & 205 & 254 & 252 \\
27 & 84 & 161 & 29 & 124 & 204 & 228 & 176 & 73 & 49 & 39 & 45 & 83 & 105 & 2 & 245 \\
24 & 223 & 68 & 79 & 155 & 188 & 15 & 92 & 11 & 220 & 189 & 148 & 172 & 9 & 199 & 162 \\
28 & 130 & 159 & 198 & 52 & 194 & 70 & 5 & 206 & 59 & 13 & 60 & 156 & 8 & 190 & 183 \\
135 & 229 & 238 & 107 & 235 & 242 & 191 & 175 & 197 & 100 & 7 & 123 & 149 & 154 & 174 & 182 \\
18 & 89 & 165 & 53 & 101 & 184 & 163 & 158 & 210 & 247 & 98 & 90 & 133 & 125 & 168 & 58 \\
41 & 113 & 200 & 246 & 249 & 67 & 215 & 214 & 16 & 115 & 118 & 120 & 153 & 10 & 25 & 145 \\
20 & 63 & 230 & 240 & 134 & 177 & 226 & 241 & 250 & 116 & 243 & 180 & 109 & 33 & 178 & 106 \\
227 & 231 & 181 & 234 & 3 & 143 & 211 & 201 & 66 & 212 & 232 & 117 & 127 & 255 & 126 & 253 \\
\hline
\end{tabular}

Table 4 . The proposed multiplicative inverse $m_{3}$

\begin{tabular}{lllllllllllllll}
\hline 0 & 1 & 149 & 230 & 223 & 187 & 115 & 164 & 250 & 133 & 200 & 85 & 172 & 206 & 82 \\
125 & 39 & 215 & 248 & 100 & 89 & 191 & 163 & 86 & 80 & 103 & 154 & 41 & 51 & 161 \\
171 & 145 & 134 & 232 & 254 & 225 & 124 & 17 & 50 & 28 & 185 & 48 & 202 & 118 & 196 \\
43 & 184 & 40 & 29 & 166 & 177 & 77 & 63 & 129 & 97 & 140 & 90 & 197 & 47 & 76 \\
192 & 244 & 221 & 68 & 67 & 220 & 116 & 252 & 127 & 143 & 229 & 198 & 62 & 54 & 157 \\
25 & 87 & 14 & 104 & 201 & 11 & 24 & 81 & 101 & 21 & 59 & 141 & 98 & 151 & 139 \\
128 & 57 & 92 & 150 & 20 & 88 & 155 & 26 & 83 & 15 & 205 & 217 & 179 & 158 & 138 \\
213 & 242 & 165 & 6 & 70 & 253 & 45 & 203 & 247 & 226 & 130 & 237 & 38 & 16 & 142 \\
96 & 56 & 122 & 236 & 251 & 9 & 34 & 233 & 180 & 194 & 110 & 94 & 58 & 91 & 126 \\
170 & 33 & 210 & 183 & 231 & 2 & 99 & 93 & 31 & 160 & 27 & 102 & 219 & 78 & 109 \\
153 & 30 & 190 & 23 & 7 & 114 & 52 & 176 & 241 & 239 & 144 & 32 & 12 & 207 & 189 \\
167 & 53 & 159 & 108 & 136 & 195 & 211 & 147 & 49 & 42 & 222 & 5 & 208 & 174 & 162 \\
64 & 245 & 137 & 181 & 46 & 60 & 75 & 228 & 10 & 84 & 44 & 119 & 216 & 106 & 13 \\
188 & 175 & 146 & 182 & 243 & 112 & 249 & 18 & 204 & 107 & 79 & 156 & 69 & 66 & 186 \\
255 & 37 & 121 & 246 & 199 & 74 & 3 & 148 & 35 & 135 & 235 & 234 & 131 & 123 & 240 \\
238 & 168 & 113 & 212 & 65 & 193 & 227 & 120 & 19 & 214 & 8 & 132 & 71 & 117 & 36 \\
\hline
\end{tabular}

In the same way, it can be constructed S-box 4, S-box 5, S-box 6 , S-box 7, S-box 8, S-box 9

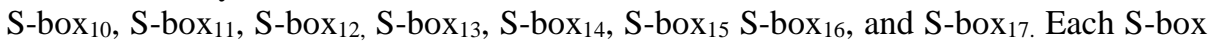
produced will be tested using SAC. SAC is defined in the following Eq. (2) [3]:

$$
S(x)=\left(\frac{1}{2^{n}} \sum_{i=1}^{n} f(x) \oplus f\left(x \oplus c_{i}^{n}\right)\right)
$$

where $n$ is the number of variables, and $i$ is a 1-bit value in position $i$ th. In detail, the process of calculating SAC value in $\mathrm{S}^{-b_{0}}{ }_{1}$ is illustrated in Tables 8, 9, and 10. 
Table 5. The proposed S-box 1

\begin{tabular}{lllllllllllllll}
\hline 99 & 124 & 119 & 123 & 242 & 107 & 111 & 197 & 48 & 1 & 103 & 43 & 254 & 215 & 171 \\
202 & 130 & 201 & 125 & 250 & 89 & 71 & 240 & 173 & 212 & 162 & 175 & 156 & 164 & 114 \\
183 & 253 & 147 & 38 & 54 & 63 & 247 & 204 & 52 & 165 & 229 & 241 & 113 & 216 & 49 \\
4 & 199 & 35 & 195 & 24 & 150 & 5 & 154 & 7 & 18 & 128 & 226 & 235 & 39 & 178 \\
9 & 131 & 44 & 26 & 27 & 110 & 90 & 160 & 82 & 59 & 214 & 179 & 41 & 227 & 47 \\
83 & 209 & 0 & 237 & 32 & 252 & 177 & 91 & 106 & 203 & 190 & 57 & 74 & 76 & 88 \\
208 & 239 & 170 & 251 & 67 & 77 & 51 & 133 & 69 & 249 & 2 & 127 & 80 & 60 & 159 \\
81 & 163 & 64 & 143 & 146 & 157 & 56 & 245 & 188 & 182 & 218 & 33 & 16 & 255 & 243 \\
205 & 12 & 19 & 236 & 95 & 151 & 68 & 23 & 196 & 167 & 126 & 61 & 100 & 93 & 25 \\
96 & 129 & 79 & 220 & 34 & 42 & 144 & 136 & 70 & 238 & 184 & 20 & 222 & 94 & 11 \\
224 & 50 & 58 & 10 & 73 & 6 & 36 & 92 & 194 & 211 & 172 & 98 & 145 & 149 & 228 \\
231 & 200 & 55 & 109 & 141 & 213 & 78 & 169 & 108 & 86 & 244 & 234 & 101 & 122 & 174 \\
186 & 120 & 37 & 46 & 28 & 166 & 180 & 198 & 232 & 221 & 116 & 31 & 75 & 189 & 139 \\
112 & 62 & 181 & 102 & 72 & 3 & 246 & 14 & 97 & 53 & 87 & 185 & 134 & 193 & 29 \\
225 & 248 & 152 & 17 & 105 & 217 & 142 & 148 & 155 & 30 & 135 & 233 & 206 & 85 & 40 \\
140 & 161 & 137 & 13 & 191 & 230 & 66 & 104 & 65 & 153 & 45 & 15 & 176 & 84 & 187 \\
\hline
\end{tabular}

Table 6. The proposed S-box 2

\begin{tabular}{lllllllllllllll}
\hline 99 & 124 & 86 & 69 & 249 & 82 & 112 & 56 & 148 & 134 & 65 & 229 & 234 & 201 & 206 \\
34 & 136 & 43 & 173 & 200 & 203 & 32 & 5 & 29 & 96 & 54 & 236 & 15 & 205 & 125 \\
195 & 83 & 150 & 74 & 71 & 23 & 4 & 42 & 182 & 218 & 55 & 98 & 194 & 53 & 80 \\
92 & 11 & 226 & 58 & 115 & 10 & 164 & 239 & 85 & 190 & 142 & 232 & 214 & 231 & 241 \\
51 & 240 & 193 & 185 & 35 & 30 & 77 & 31 & 113 & 20 & 89 & 40 & 208 & 26 & 199 \\
137 & 161 & 191 & 104 & 243 & 84 & 227 & 88 & 179 & 52 & 242 & 102 & 64 & 217 & 44 \\
252 & 8 & 237 & 140 & 25 & 87 & 207 & 63 & 107 & 119 & 109 & 223 & 128 & 76 & 37 \\
120 & 197 & 141 & 61 & 47 & 224 & 28 & 81 & 3 & 235 & 33 & 39 & 144 & 176 & 131 \\
75 & 41 & 16 & 9 & 50 & 175 & 180 & 254 & 67 & 110 & 221 & 27 & 116 & 160 & 93 \\
106 & 127 & 216 & 1 & 196 & 122 & 198 & 209 & 186 & 94 & 101 & 97 & 139 & 132 & 118 \\
22 & 210 & 184 & 105 & 13 & 21 & 230 & 0 & 145 & 168 & 248 & 245 & 153 & 155 & 68 \\
177 & 171 & 114 & 158 & 17 & 7 & 91 & 170 & 72 & 59 & 62 & 111 & 126 & 219 & 181 \\
172 & 178 & 108 & 18 & 36 & 6 & 46 & 167 & 228 & 100 & 121 & 147 & 143 & 45 & 247 \\
103 & 169 & 211 & 123 & 222 & 133 & 135 & 152 & 146 & 151 & 244 & 78 & 250 & 165 & 117 \\
238 & 212 & 138 & 57 & 174 & 225 & 246 & 38 & 255 & 202 & 24 & 130 & 220 & 159 & 192 \\
233 & 149 & 157 & 14 & 66 & 73 & 251 & 204 & 154 & 166 & 48 & 213 & 19 & 156 & 12 \\
\hline
\end{tabular}

Table 7. The proposed S-box 3

\begin{tabular}{llllllllllllll}
\hline 99 & 124 & 126 & 138 & 127 & 39 & 151 & 115 & 255 & 143 & 211 & 54 & 139 & 145 \\
45 & 221 & 135 & 193 & 59 & 178 & 91 & 46 & 23 & 85 & 26 & 219 & 103 & 80 \\
214 & 2 & 174 & 48 & 131 & 215 & 50 & 141 & 79 & 22 & 25 & 113 & 237 & 244 \\
89 & 6 & 120 & 9 & 77 & 225 & 63 & 212 & 243 & 88 & 104 & 147 & 72 & 37 \\
43 & 69 & 65 & 216 & 133 & 94 & 202 & 189 & 19 & 73 & 171 & 105 & 203 & 51 \\
117 & 8 & 217 & 191 & 204 & 186 & 106 & 74 & 36 & 241 & 168 & 119 & 121 & 64 \\
236 & 150 & 209 & 95 & 238 & 173 & 196 & 84 & 116 & 198 & 176 & 61 & 223 & 167 \\
185 & 7 & 108 & 33 & 230 & 162 & 27 & 242 & 100 & 246 & 210 & 83 & 194 & 146 \\
71 & 137 & 112 & 76 & 224 & 132 & 190 & 47 & 130 & 21 & 253 & 239 & 183 & 140 \\
201 & 159 & 228 & 163 & 149 & 93 & 102 & 206 & 55 & 15 & 75 & 5 & 3 & 30 \\
250 & 40 & 68 & 207 & 62 & 136 & 13 & 254 & 38 & 109 & 29 & 128 & 231 & 142 \\
82 & 18 & 184 & 195 & 20 & 10 & 251 & 60 & 110 & 70 & 96 & 0 & 218 & 181 \\
164 & 90 & 11 & 157 & 58 & 245 & 125 & 180 & 165 & 41 & 4 & 235 & 34 & 129 \\
122 & 170 & 35 & 188 & 24 & 182 & 222 & 172 & 175 & 158 & 1 & 153 & 199 & 154
\end{tabular}


Table 8. Bit input S-box 1

\begin{tabular}{llllllll}
\hline 0 & 0 & 0 & 0 & 0 & 0 & 0 & 0 \\
0 & 0 & 0 & 0 & 0 & 0 & 0 & 1 \\
0 & 0 & 0 & 0 & 0 & 0 & 1 & 0 \\
0 & 0 & 0 & 0 & 0 & 0 & 1 & 1 \\
0 & 0 & 0 & 0 & 0 & 1 & 0 & 0 \\
0 & 0 & 0 & 0 & 0 & 1 & 0 & 1 \\
0 & 0 & 0 & 0 & 0 & 1 & 1 & 0 \\
0 & 0 & 0 & 0 & 0 & 1 & 1 & 1 \\
0 & 0 & 0 & 0 & 1 & 0 & 0 & 0 \\
0 & 0 & 0 & 0 & 1 & 0 & 0 & 1 \\
0 & 0 & 0 & 0 & 1 & 0 & 1 & 0 \\
0 & 0 & 0 & 0 & 1 & 0 & 1 & 1 \\
0 & 0 & 0 & 0 & 1 & 1 & 0 & 0 \\
0 & 0 & 0 & 0 & 1 & 1 & 0 & 1 \\
0 & 0 & 0 & 0 & 1 & 1 & 1 & 0 \\
0 & 0 & 0 & 0 & 1 & 1 & 1 & 1 \\
0 & 0 & 0 & 1 & 0 & 0 & 0 & 0 \\
0 & 0 & 0 & 1 & 0 & 0 & 0 & 1 \\
0 & 0 & 0 & 1 & 0 & 0 & 1 & 0 \\
0 & 0 & 0 & 1 & 0 & 0 & 1 & 1 \\
0 & 0 & 0 & 1 & 0 & 1 & 0 & 0 \\
0 & 0 & 0 & 1 & 0 & 1 & 0 & 1 \\
0 & 0 & 0 & 1 & 0 & 1 & 1 & 0 \\
0 & 0 & 0 & 1 & 0 & 1 & 1 & 1 \\
0 & 0 & 0 & 1 & 1 & 0 & 0 & 0 \\
0 & 0 & 0 & 1 & 1 & 0 & 0 & 1 \\
0 & 0 & 0 & 1 & 1 & 0 & 1 & 0 \\
0 & 0 & 0 & 1 & 1 & 0 & 1 & 1 \\
0 & 0 & 0 & 1 & 1 & 1 & 0 & 0 \\
0 & 0 & 0 & 1 & 1 & 1 & 0 & 1 \\
0 & 0 & 0 & 1 & 1 & 1 & 1 & 0 \\
0 & 0 & 0 & 1 & 1 & 1 & 1 & 1 \\
. &. &. &. &. &. &. &. \\
. &. &. &. &. &. &. &. \\
. &. &. &. &. &. &. &. \\
1 & 1 & 1 & 1 & 1 & 0 & 0 & 0 \\
1 & 1 & 1 & 1 & 1 & 0 & 0 & 1 \\
1 & 1 & 1 & 1 & 1 & 0 & 1 & 0 \\
1 & 1 & 1 & 1 & 1 & 0 & 1 & 1 \\
1 & 1 & 1 & 1 & 1 & 1 & 0 & 0 \\
1 & 1 & 1 & 1 & 1 & 1 & 0 & 1 \\
1 & 1 & 1 & 1 & 1 & 1 & 1 & 0 \\
1 & 1 & 1 & 1 & 1 & 1 & 1 & 1 \\
\hline & & & & & & &
\end{tabular}

Table 9. Bit output S-box 1

\begin{tabular}{cccccccc}
\hline 0 & 1 & 1 & 0 & 0 & 0 & 1 & 1 \\
0 & 1 & 1 & 1 & 1 & 1 & 0 & 0 \\
0 & 1 & 1 & 1 & 0 & 1 & 1 & 1 \\
0 & 1 & 1 & 1 & 1 & 0 & 1 & 1 \\
1 & 1 & 1 & 1 & 0 & 0 & 1 & 0 \\
0 & 1 & 1 & 0 & 1 & 0 & 1 & 1 \\
0 & 1 & 1 & 0 & 1 & 1 & 1 & 1 \\
1 & 1 & 0 & 0 & 0 & 1 & 0 & 1 \\
0 & 0 & 1 & 1 & 0 & 0 & 0 & 0 \\
0 & 0 & 0 & 0 & 0 & 0 & 0 & 1 \\
0 & 1 & 1 & 0 & 0 & 1 & 1 & 1 \\
0 & 0 & 1 & 0 & 1 & 0 & 1 & 1 \\
1 & 1 & 1 & 1 & 1 & 1 & 1 & 0 \\
1 & 1 & 0 & 1 & 0 & 1 & 1 & 1 \\
1 & 0 & 1 & 0 & 1 & 0 & 1 & 1 \\
0 & 1 & 1 & 1 & 0 & 1 & 1 & 0 \\
1 & 1 & 0 & 0 & 1 & 0 & 1 & 0 \\
1 & 0 & 0 & 0 & 0 & 0 & 1 & 0 \\
1 & 1 & 0 & 0 & 1 & 0 & 0 & 1 \\
0 & 1 & 1 & 1 & 1 & 1 & 0 & 1 \\
1 & 1 & 1 & 1 & 1 & 0 & 1 & 0 \\
0 & 1 & 0 & 1 & 1 & 0 & 0 & 1 \\
0 & 1 & 0 & 0 & 0 & 1 & 1 & 1 \\
1 & 1 & 1 & 1 & 0 & 0 & 0 & 0 \\
1 & 0 & 1 & 0 & 1 & 1 & 0 & 1 \\
1 & 1 & 0 & 1 & 0 & 1 & 0 & 0 \\
1 & 0 & 1 & 0 & 0 & 0 & 1 & 0 \\
1 & 0 & 1 & 0 & 1 & 1 & 1 & 1 \\
1 & 0 & 0 & 1 & 1 & 1 & 0 & 0 \\
1 & 0 & 1 & 0 & 0 & 1 & 0 & 0 \\
0 & 1 & 1 & 1 & 0 & 0 & 1 & 0 \\
1 & 1 & 0 & 0 & 0 & 0 & 0 & 0 \\
. &. &. &. &. &. &. &. \\
. &. &. &. &. &. &. &. \\
. &. &. &. &. &. &. &. \\
0 & 1 & 0 & 0 & 0 & 0 & 0 & 1 \\
1 & 0 & 0 & 1 & 1 & 0 & 0 & 1 \\
0 & 0 & 1 & 0 & 1 & 1 & 0 & 1 \\
0 & 0 & 0 & 0 & 1 & 1 & 1 & 1 \\
1 & 0 & 1 & 1 & 0 & 0 & 0 & 0 \\
0 & 1 & 0 & 1 & 0 & 1 & 0 & 0 \\
1 & 0 & 1 & 1 & 1 & 0 & 1 & 1 \\
0 & 0 & 0 & 1 & 0 & 1 & 1 & 0 \\
\hline & & & & & & &
\end{tabular}


Table 10. Values of the first row SAC matrix S-box 1

\begin{tabular}{llllllll}
\hline 0 & 0 & 0 & 1 & 1 & 1 & 1 & 1 \\
0 & 0 & 0 & 1 & 1 & 1 & 1 & 1 \\
0 & 0 & 0 & 0 & 1 & 1 & 0 & 0 \\
0 & 0 & 0 & 0 & 1 & 1 & 0 & 0 \\
1 & 0 & 0 & 1 & 1 & 0 & 0 & 1 \\
1 & 0 & 0 & 1 & 1 & 0 & 0 & 1 \\
1 & 0 & 1 & 0 & 1 & 0 & 1 & 0 \\
1 & 0 & 1 & 0 & 1 & 0 & 1 & 0 \\
0 & 0 & 1 & 1 & 0 & 0 & 0 & 1 \\
0 & 0 & 1 & 1 & 0 & 0 & 0 & 1 \\
0 & 1 & 0 & 0 & 1 & 1 & 0 & 0 \\
0 & 1 & 0 & 0 & 1 & 1 & 0 & 0 \\
0 & 0 & 1 & 0 & 1 & 0 & 0 & 1 \\
0 & 0 & 1 & 0 & 1 & 0 & 0 & 1 \\
1 & 1 & 0 & 1 & 1 & 1 & 0 & 1 \\
1 & 1 & 0 & 1 & 1 & 1 & 0 & 1 \\
0 & 1 & 0 & 0 & 1 & 0 & 0 & 0 \\
0 & 1 & 0 & 0 & 1 & 0 & 0 & 0 \\
1 & 0 & 1 & 1 & 0 & 1 & 0 & 0 \\
1 & 0 & 1 & 1 & 0 & 1 & 0 & 0 \\
1 & 0 & 1 & 0 & 0 & 0 & 1 & 1 \\
1 & 0 & 1 & 0 & 0 & 0 & 1 & 1 \\
1 & 0 & 1 & 1 & 0 & 1 & 1 & 1 \\
1 & 0 & 1 & 1 & 0 & 1 & 1 & 1 \\
0 & 1 & 1 & 1 & 1 & 0 & 0 & 1 \\
0 & 1 & 1 & 1 & 1 & 0 & 0 & 1 \\
0 & 0 & 0 & 0 & 1 & 1 & 0 & 1 \\
0 & 0 & 0 & 0 & 1 & 1 & 0 & 1 \\
0 & 0 & 1 & 1 & 1 & 0 & 0 & 0 \\
0 & 0 & 1 & 1 & 1 & 0 & 0 & 0 \\
1 & 0 & 1 & 1 & 0 & 0 & 1 & 0 \\
1 & 0 & 1 & 1 & 0 & 0 & 1 & 0 \\
. &. &. &. &. &. &. &. \\
. &. &. &. &. &. &. &. \\
. &. &. &. &. &. &. &. \\
1 & 1 & 0 & 1 & 1 & 0 & 0 & 0 \\
1 & 1 & 0 & 1 & 1 & 0 & 0 & 0 \\
0 & 0 & 1 & 0 & 0 & 0 & 1 & 0 \\
0 & 0 & 1 & 0 & 0 & 0 & 1 & 0 \\
1 & 1 & 1 & 0 & 0 & 1 & 0 & 0 \\
1 & 1 & 1 & 0 & 0 & 1 & 0 & 0 \\
1 & 0 & 1 & 0 & 1 & 1 & 0 & 1 \\
1 & 0 & 1 & 0 & 1 & 1 & 0 & 1 \\
\hline 128 & 116 & 124 & 116 & 144 & 116 & 132 & 132
\end{tabular}




\section{RESULT AND DISCUSSION}

The number of variables in $\mathrm{GF}\left(2^{8}\right)$ is 8 . The values of $\sum_{i=1}^{n} f(x) \oplus f\left(x \oplus c_{i}^{n}\right)$ are 128, $116,124,116,144,116,132$, and 132. According to Eq. (2), the values of the SAC matrix produced by the first row are $0.5,0.453125,0.484375,0.453125,0.5625$,

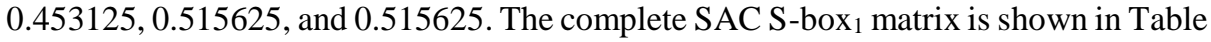
11. Based on Table 11, the average $S A C$ value in $S-b_{0 x}$ is 0.50488 . The average values of SAC in other proposed S-boxes, i.e, S-box 2, S-box 3 , S-box $4, \ldots$, S-box 17 are shown in Table 12.

Table 11. The SAC matrix of the proposed S-box 1

\begin{tabular}{rrrrrrrr}
\hline 0.5 & 0.453125 & 0.484375 & 0.45313 & 0.5625 & 0.453125 & 0.515625 & 0.515625 \\
0.5313 & 0.5 & 0.453125 & 0.48438 & 0.5 & 0.5625 & 0.484375 & 0.46875 \\
0.5 & 0.53125 & 0.5 & 0.5625 & 0.46875 & 0.5 & 0.515625 & 0.515625 \\
0.5469 & 0.5 & 0.53125 & 0.5 & 0.453125 & 0.46875 & 0.53125 & 0.53125 \\
0.5313 & 0.546875 & 0.5 & 0.5 & 0.515625 & 0.453125 & 0.5 & 0.453125 \\
0.5313 & 0.53125 & 0.546875 & 0.46875 & 0.46875 & 0.515625 & 0.515625 & 0.453125 \\
0.4844 & 0.53125 & 0.53125 & 0.46875 & 0.515625 & 0.46875 & 0.53125 & 0.53125 \\
0.5156 & 0.484375 & 0.53125 & 0.48438 & 0.53125 & 0.515625 & 0.5625 & 0.515625 \\
\hline \hline
\end{tabular}

Table 12. The SAC mean values of the proposed S-box 1 to S-box 17

\begin{tabular}{|c|c|}
\hline S-boxes & SAC \\
\hline Proposed S-box 1 & 0.50488 \\
\hline Proposed S-box2 & 0.49658 \\
\hline Proposed S-box3 & 0.50635 \\
\hline Proposed S-box 4 & 0.51343 \\
\hline Proposed S-box 5 & 0.50806 \\
\hline Proposed S-box6 & 0.49585 \\
\hline Proposed S-box7 & 0.49951 \\
\hline Proposed S-box8 & 0.50757 \\
\hline Proposed S-box9 & 0.51025 \\
\hline Proposed S-box 10 & 0.50073 \\
\hline Proposed S-box 11 & 0.49780 \\
\hline Proposed S-box 12 & 0.49780 \\
\hline Proposed S-box 13 & 0.50781 \\
\hline Proposed S-box 14 & 0.50220 \\
\hline Proposed S-box 15 & 0.49292 \\
\hline Proposed S-box 16 & 0.50757 \\
\hline Proposed S-box 17 & 0.50000 \\
\hline
\end{tabular}

According to Table 12, the perfect SAC value is found in the proposed S-box ${ }_{17}$ with a value of 0.5 . Thus, the best $S$-box is the proposed $S$-box ${ }_{17}$. The multiplicative inverse table $m_{17}$ and proposed S-box 17 are shown in Tables 13 and 14. Table 15 shows the 
values of the SAC matrix from the proposed S-box ${ }_{17}$. In the next section, we will discuss the SAC performance analysis of each S-box produced in previous studies that will be compared with the best S-box, i.e., proposed S-box 17 .

Table 13. The proposed multiplicative inverse $m_{17}$

\begin{tabular}{llllllllllllllll}
\hline 0 & 1 & 225 & 190 & 145 & 106 & 95 & 129 & 169 & 127 & 53 & 101 & 206 & 83 & 161 & 38 \\
181 & 163 & 222 & 30 & 251 & 118 & 211 & 49 & 103 & 208 & 200 & 148 & 177 & 35 & 19 & 223 \\
187 & 244 & 176 & 29 & 111 & 108 & 15 & 160 & 156 & 93 & 59 & 241 & 136 & 205 & 249 & 195 \\
210 & 23 & 104 & 97 & 100 & 10 & 74 & 218 & 185 & 174 & 240 & 42 & 232 & 132 & 142 & 147 \\
188 & 113 & 122 & 139 & 88 & 246 & 239 & 124 & 214 & 221 & 54 & 219 & 230 & 167 & 80 & 170 \\
78 & 171 & 207 & 13 & 252 & 158 & 153 & 114 & 68 & 247 & 135 & 165 & 157 & 41 & 128 & 6 \\
105 & 51 & 234 & 237 & 52 & 11 & 209 & 24 & 50 & 96 & 5 & 144 & 37 & 110 & 109 & 36 \\
189 & 65 & 87 & 152 & 120 & 196 & 21 & 250 & 116 & 197 & 66 & 138 & 71 & 238 & 168 & 9 \\
94 & 7 & 217 & 242 & 61 & 233 & 164 & 90 & 44 & 204 & 123 & 67 & 150 & 199 & 62 & 146 \\
107 & 4 & 143 & 63 & 27 & 201 & 140 & 198 & 115 & 86 & 178 & 183 & 40 & 92 & 85 & 253 \\
39 & 14 & 180 & 17 & 134 & 91 & 231 & 77 & 126 & 8 & 79 & 81 & 173 & 172 & 57 & 184 \\
34 & 28 & 154 & 182 & 162 & 16 & 179 & 155 & 175 & 56 & 245 & 32 & 64 & 112 & 3 & 224 \\
213 & 226 & 248 & 47 & 117 & 121 & 151 & 141 & 26 & 149 & 228 & 255 & 137 & 45 & 12 & 82 \\
25 & 102 & 48 & 22 & 227 & 192 & 72 & 220 & 243 & 130 & 55 & 75 & 215 & 73 & 18 & 31 \\
191 & 2 & 193 & 212 & 202 & 254 & 76 & 166 & 60 & 133 & 98 & 236 & 235 & 99 & 125 & 70 \\
58 & 43 & 131 & 216 & 33 & 186 & 69 & 89 & 194 & 46 & 119 & 20 & 84 & 159 & 229 & 203 \\
\hline
\end{tabular}

Table 14. The proposed S-box 17

\begin{tabular}{llllllllllllllll}
\hline 99 & 124 & 215 & 68 & 2 & 129 & 240 & 243 & 232 & 19 & 18 & 36 & 145 & 116 & 16 & 194 \\
157 & 46 & 96 & 40 & 224 & 244 & 251 & 110 & 26 & 218 & 211 & 97 & 225 & 161 & 179 & 127 \\
39 & 69 & 254 & 9 & 226 & 195 & 198 & 15 & 153 & 206 & 168 & 38 & 20 & 176 & 222 & 10 \\
228 & 207 & 191 & 88 & 59 & 165 & 98 & 28 & 25 & 181 & 57 & 70 & 48 & 144 & 86 & 60 \\
122 & 169 & 112 & 53 & 173 & 123 & 109 & 50 & 152 & 65 & 51 & 3 & 138 & 82 & 85 & 201 \\
30 & 214 & 142 & 248 & 189 & 167 & 250 & 136 & 216 & 100 & 177 & 108 & 134 & 103 & 236 & 33 \\
160 & 80 & 14 & 83 & 13 & 186 & 197 & 106 & 79 & 71 & 0 & 29 & 227 & 253 & 220 & 252 \\
101 & 187 & 8 & 229 & 78 & 87 & 241 & 255 & 202 & 72 & 154 & 42 & 249 & 114 & 247 & 132 \\
239 & 62 & 61 & 7 & 234 & 47 & 115 & 147 & 4 & 175 & 111 & 133 & 95 & 118 & 203 & 35 \\
158 & 31 & 73 & 212 & 75 & 204 & 104 & 105 & 151 & 23 & 192 & 163 & 120 & 209 & 54 & 162 \\
221 & 217 & 130 & 141 & 174 & 140 & 149 & 63 & 12 & 155 & 1 & 74 & 148 & 139 & 150 & 6 \\
190 & 22 & 219 & 188 & 49 & 146 & 223 & 196 & 170 & 137 & 90 & 128 & 164 & 182 & 66 & 200 \\
185 & 246 & 193 & 37 & 213 & 81 & 64 & 119 & 84 & 126 & 180 & 156 & 11 & 27 & 231 & 107 \\
117 & 5 & 113 & 208 & 233 & 43 & 92 & 94 & 24 & 210 & 44 & 125 & 135 & 67 & 172 & 55 \\
91 & 93 & 52 & 166 & 237 & 131 & 32 & 77 & 245 & 143 & 121 & 76 & 17 & 102 & 45 & 230 \\
183 & 89 & 205 & 34 & 159 & 56 & 199 & 178 & 21 & 58 & 235 & 238 & 41 & 184 & 171 & 242 \\
\hline \hline
\end{tabular}


Table 15. The SAC matrix of the proposed S-box 17

\begin{tabular}{rrrrrrrr}
\hline 0.5469 & 0.4531 & 0.4531 & 0.4531 & 0.4688 & 0.4531 & 0.5313 & 0.5 \\
0.5 & 0.5469 & 0.5 & 0.5156 & 0.5469 & 0.4688 & 0.5469 & 0.4375 \\
0.4375 & 0.5 & 0.4844 & 0.5 & 0.5156 & 0.5469 & 0.5156 & 0.4844 \\
0.4844 & 0.4375 & 0.5 & 0.4688 & 0.4844 & 0.5156 & 0.5156 & 0.5469 \\
0.5469 & 0.4844 & 0.5313 & 0.5156 & 0.4688 & 0.4844 & 0.4688 & 0.5 \\
0.5 & 0.5469 & 0.5469 & 0.4844 & 0.5156 & 0.4688 & 0.5156 & 0.5313 \\
0.5313 & 0.5 & 0.5313 & 0.5156 & 0.4688 & 0.5156 & 0.5469 & 0.5313 \\
0.5313 & 0.5313 & 0.4688 & 0.5 & 0.4688 & 0.4688 & 0.4375 & 0.4844 \\
\hline
\end{tabular}

\subsection{Performance Analysis of The Novel S-Boxes}

Table 16 shows the performance comparison of SAC values from S-boxes that were previously made. Based on Table 16 , the proposed $S-$ box $_{17}$ has the best SAC value of 0.5 .

Table 16. Performance comparison

\begin{tabular}{ll}
\hline S-boxes & SAC \\
\hline AES & 0.5048 \\
In [7] & 0.5107 \\
In [8] & 0.5066 \\
In [9] & 0.5064 \\
In [10] & - \\
In [11] & 0.4956 \\
In [12] & 0.4983 \\
In [13] & 0.5036 \\
In [14] & 0.4976 \\
In [15] & 0.4930 \\
In [16] & 0.4978 \\
In [17] & 0.503 \\
In [18] & 0.503 \\
In [19] & 0.504 \\
In [20] & 0.502 \\
In [21] & 0.498 \\
In [22] & 0.5002 \\
In [23] & 0.5017 \\
Proposed S-box 17 & $\mathbf{0 . 5 0 0 0}$ \\
\hline
\end{tabular}

According to the previous discussion about S-box construction, it can be concluded that the proposed S-box 17 has a perfect SAC value and is the best value compared to the Sbox results of previous studies. 


\section{CONCLUSION}

In this research, a novel method was introduced in building S-box construction to get the perfect SAC value. The S-box construction starts with classifying the irreducible polynomial based on the number of the least polynomial elements (simple irreducible polynomial). The selection of simply reduced polynomials aims to simplify calculations in building multiplicative inverse. The results of each element of the multiplication inverse are applied to the affine matrix and the addition of a constant 8-bit vector to produce an S-box. This result shows that the proposed S-box 17 has a perfect SAC value of 0.5. So it can be concluded that the proposed S-box has the best SAC value compared to the S-boxes from the previous research. For the next research, it is expected that there will be further research in S-box construction that has perfect value other than the SAC criteria in a good S-box test.

\section{REFERENCES}

[1] Wu, C. K., \& Feng, D. (2016). Boolean functions and their applications in cryptography. Springer Berlin Heidelberg.

[2] Hussain, I., \& Shah, T. (2013). Literature survey on nonlinear components and chaotic nonlinear components of block ciphers. Nonlinear Dynamics, 74(4), 869904.

[3] Daemen, J., \& Rijmen, V. (2002). The design of Rijndael (Vol. 2). New York: Springer-verlag.

[4] C. Paar and J. Pelzl. (2010). Understanding Cryptography, 1st ed., vol. 1. Springer-Verlag Berlin Heidelberg.

[5] Alamsyah, Bejo, A., \& Adji, T. B. (2017, August). AES S-box construction using different irreducible polynomial and constant 8-bit vector. In 2017 IEEE Conference on Dependable and Secure Computing (pp. 366-369). IEEE.

[6] Williams, H., Webster, A., \& Tavares, S. (1986). On the design of s-boxes. In Advances in Cryptology-CRYPTO'85 Proceedings (Vol. 218, pp. 523-534).

[7] Girija, R., \& Singh, H. (2018). Enhancing security of double random phase encoding based on random S-Box. 3D Research, 9(2), 15.

[8] Farwa, S., Muhammad, N., Shah, T., \& Ahmad, S. (2017). A novel image encryption based on algebraic S-box and Arnold transform. 3D Research, 8(3), 26.

[9] Çavuşoğlu, Ü., Kaçar, S., Pehlivan, I., \& Zengin, A. (2017). Secure image encryption algorithm design using a novel chaos based S-Box. Chaos, Solitons \& Fractals, 95, 92-101.

[10] Hussain, I., Anees, A., AlKhaldi, A. H., Algarni, A., \& Aslam, M. (2018). Construction of chaotic quantum magnets and matrix Lorenz systems S-boxes and their applications. Chinese Journal of Physics, 56(4), 1609-1621.

[11] Belazi, A., Khan, M., El-Latif, A. A. A., \& Belghith, S. (2017). Efficient cryptosystem approaches: S-boxes and permutation-substitution-based encryption. Nonlinear Dynamics, 87(1), 337-361.

[12] Özkaynak, F., Çelik, V., \& Özer, A. B. (2017). A new S-box construction method based on the fractional-order chaotic Chen system. Signal, Image and Video Processing, 11(4), 659-664.

[13] Özkaynak, F., \& Yavuz, S. (2013). Designing chaotic S-boxes based on timedelay chaotic system. Nonlinear Dynamics, 74(3), 551-557. 
[14] Liu, G., Yang, W., Liu, W., \& Dai, Y. (2015). Designing S-boxes based on 3-D four-wing autonomous chaotic system. Nonlinear Dynamics, 82(4), 1867-1877.

[15] Khan, M., Shah, T., Mahmood, H., Gondal, M. A., \& Hussain, I. (2012). A novel technique for the construction of strong S-boxes based on chaotic Lorenz systems. Nonlinear Dynamics, 70(3), 2303-2311.

[16] Khan, M., \& Shah, T. (2015). An efficient construction of substitution box with the fractional chaotic system. Signal, Image, and Video Processing, 9(6), 13351338.

[17] Lambić, D. (2017). A novel method of S-box design based on a discrete chaotic map. Nonlinear Dynamics, 87(4), 2407-2413.

[18] Khan, M., \& Azam, N. A. (2015). S-boxes based on affine mapping and orbit of power function. 3D Research, 6(2), 12.

[19] Çavuşoğlu, Ü., Zengin, A., Pehlivan, I., \& Kaçar, S. (2017). A novel approach for strong S-Box generation algorithm design based on chaotic scaled Zhongtang system. Nonlinear Dynamics, 87(2), 1081-1094.

[20] Ullah, A., Jamal, S. S., \& Shah, T. (2017). A novel construction of substitution box using a combination of chaotic maps with improved chaotic range. Nonlinear Dynamics, 88(4), 2757-2769.

[21] Isa, H., Jamil, N., \& Z'aba, M. R. (2016). Construction of cryptographically strong S-Boxes inspired by bee waggle dance. New generation computing, 34(3), 221238.

[22] Ul Islam, F., \& Liu, G. (2017). Designing S-box based on 4D-4wing hyperchaotic system. 3D Research, 8(1), 9.

[23] Hussain, I., Gondal, M. A., \& Hussain, A. (2015). Construction of Substitution Box Based on Piecewise Linear Chaotic Map and S 8 Group. 3D Research, 6(1), 3.

[24] Stallings, W. (2014). Cryptography and network security: principles and practice, international edition: principles and practice. Pearson Higher Ed.

[25] Gangadari, B. R., \& Ahamed, S. R. (2015, August). Analysis and algebraic construction of S-Box for AES algorithm using irreducible polynomials. In 2015 Eighth International Conference on Contemporary Computing (IC3) (pp. 526530). IEEE.

[26] Wang, D., \& Sun, S. L. (2008, December). Replacement and Structure of S-boxes in Rijndael. In 2008 International Conference on Computer Science and Software Engineering (Vol. 3, pp. 782-784). IEEE.

[27] Alamsyah, Bejo, A., \& Adji, T. B. (2018). The replacement of irreducible polynomial and affine mapping for the construction of a strong S-box. Nonlinear Dynamics, 93(4), 2105-2118.

[28] Alamsyah, Bejo, A., \& Adji, T. B. (2018, October). S-box Construction of Highly Strict Avalanche Criterion Using Algebraic Technique. In 2018 Third International Conference on Informatics and Computing (ICIC) (pp. 1-4). IEEE.

[29] Alamsyah, Bejo, A., \& Adji, T. B. (2019, October). Enhancement strict avalanche criterion value in robust $\mathrm{S}$-boxes construction using selected irreducible polynomial and affine matrixes. In Journal of Physics: Conference Series (Vol. 1321, No. 3, p. 032020). IOP Publishing. 